\title{
Coronary Interventions with Mechanical Circulatory Support
}

\author{
Markus Ferrari \\ University Heart Centre Thuringia, Jena, \\ Germany
}

\section{Introduction}

Patients in cardiogenic shock, with severely impaired left ventricular function, or intervention on the last open supplying coronary artery are summarized as so called "high risk coronary interventions" with significant risk of hemodynamic collapse and increased periprocedural mortality rate [1]. Thus, high risk percutaneous coronary interventions (PCI) may require prophylactic or standby hemodynamic mechanical circulatory support. While infusion of inotropic substances further increases myocardial oxygen consumption, other measures of support may be favourable especially in infarct related acute pump failure.

However, in acute cardiogenic shock, mechanical circulatory support may depict the only option to stabilise the patient for intra- or inter-hospital transfer and further diagnostic and therapeutic measures. This chapter summarises different therapeutic options of mechanical circulatory support in high risk PCI patients. Ongoing technical development as well as clinical studies may further elucidate this field of interventional cardiology in the future.

\section{Identification of high risk $\mathrm{PCl}$ patients and indication for mechanical circulatory support}

Cardiogenic shock is defined as reduction of cardiac index to less than $2.21 / \mathrm{min} / \mathrm{m}^{2}$ and increase in pulmonary capillary wedge pressure above $18 \mathrm{mmHg}$. Patients present with systolic blood pressure below $100 \mathrm{mmHg}$, tachycardia of more than $100 \mathrm{bpm}$, oliguria or anuria, cold and pale extremities, and impairment of cognitive function. The infusion of volume does not result in termination of shock. One-month-mortality rates of cardiogenic shock range between $40 \%$ and $80 \%$. Cardiogenic shock can be caused by multiple reasons (table 1). However, acute myocardial infarction (AMI) accounts for more than $80 \%$ of cases. Fastest possible coronary revascularisation is recommended in patients suffering from AMI related cardiogenic shock [2].

Some cardiogenic shock patients with ongoing CPR require mechanical circulatory support upfront to make coronary angiography and/or PCI feasible. Besides catecholamine infusion, mechanical circulatory support provides an increase in arterial blood pressure and therefore in organ perfusion resulting in an improvement of organ function. The prognosis of shock patients is determined by the occurrence of a multi organ dysfunction syndrome (MODS) associated with an extremely high mortality. Hemodynamic support should increase arterial blood pressure to values above $70 \mathrm{mmHg}$ for prevention or reversal of beginning MODS. 


\begin{tabular}{|l|ll|}
\hline & - & Myocardial ischemia, myocardial infarction \\
& - & Myocarditis \\
Myocardial & - & Dilatated cardiomyopathy \\
pump failure & - & Hypertrophic or restrictive cardiomyopathy \\
& - & Takotsubo-Syndrome \\
& - & Cardiotoxic substances (intoxication, cytostatics, drugs) \\
& & Cardiodepressive substances (B-blockers, Ca-antagonists, drugs, \\
Rhythm related & - & Supra-ventricular tachycardia or bradycardia \\
pump failure & - & Conductance disorders (AV-block, pre-excitation) \\
& - & Asystoly, electro-mechanical discordance \\
\hline & - & Valvular heart disease (stenosis, insufficiency) \\
Mechanical & - & Mechanical complications of infarction (rupture of papillary \\
problems & - & Cardiac tamponade \\
& - & Pericarditis constrictiva \\
& - & Intra-cavity thrombus \\
& - & Hypertrophic obstructive cardiomyopathy \\
& - & Aortic dissection
\end{tabular}

Table 1. Reasons of acute cardiogenic shock

Three groups of patients requiring mechanical circulatory support in the catheter laboratory can be identified:

1. Acute pump failure or circulatory arrest with cardiogenic shock.

2. Elective high risk PCI patients requiring prophylactic or standby support if the risk for hemodynamic collapse is relatively high.

3. Effective hemodynamic stabilisation for transfer of shock patients to other departments or other heart centres (e.g. cardiac surgery).

While patients in group 1 and 3 can be easily identified by clinical judgement and hemodynamic evaluation, the indication for mechanical support in high risk elective PCI is still based on individual judgement, although scoring systems like Jeopardy Score or Bergelson's Score may help in the clinical process of decision making [3]. While elective circulatory support can be recommended in patients with severely impaired left ventricular ejection fraction, the standby of assist devices is also an option in hemodynamically stable patients.

High risk PCI is defined as PCI of target vessel supplying more than $50 \%$ of vital myocardium, or as PCI in unstable patients with an impaired left ventricular ejection fraction below 25\% [1]. Several scoring systems may help to identify high risk PCI candidates based on the coronary anatomy. The Jeopardy Score introduced by Califf et al. in 1988 divides coronary circulation in six major areas. In case of occlusion or severe stenosis each segment accounts one point, in case of akinesia in the area only 0.5 points. If this analysis of the coronary tree adds to more than 3 points the patient is endangered for hemodynamic collapse during PCI [4]. However, all scoring systems origin from data bases of the late 80's and 90's when direct stenting and rapid exchange coronary devices were not available in the same extend as today. Due to lack of validated scoring systems, the application of surgical scoring systems like STS- or revised Euro-Score may support the 
process of clinical decision making for use of circulatory support in high risk PCI. Recent studies suggested a benefit of prophylactic circulatory support with the Impella ${ }^{\mathrm{TM}}$ in comparison to an intra aortic balloon pump (IABP) in elective high risk patients [5]. However, this study had relatively high 30-day event rates (Impella 15.3\%, IABP 21.3\%), which requests for further clinical trials. The optimal decision is mostly based on the individual situation of the patient and the experience of the operator.

\section{Mechanical circulatory support devices in the catheter laboratory}

\subsection{Intra aortic balloon pump (IABP)}

The concept of intra aortic counter pulsation was already introduced in the 50 's by the Kantrowitz brothers [6]. They showed an improvement in coronary blood flow when offering a delayed blood pressure pulse to the coronary circulation. Further technical developments included electrical stimulation of muscles wrapped around the abdominal aorta until the final design of an intra aortic balloon with a synchronised counter pulsation was introduced in cardiac surgery [7]. In the 70's, IABP use increased for treatment of acute myocardial infarction and cardiogenic shock. In case of thrombolytic therapy, IABP significantly improved survival as described in the TIMI-18 study [8]. Today, use of IABP in infarct related cardiogenic shock after successful PCI is under controversial discussion [9]. However, IABP is recommended in cardiogenic shock before and after cardiac surgery, and in acute AMI patients before coronary revascularisation to improve hemodynamic situation. An IABP consists of a helium filled balloon in the descending aorta which is inflated and deflated in a counter pulsating way to the contractions of the left ventricle. Coronary and cerebral diastolic perfusion is increased by abrupt inflation in the early diastolic phase, increasing diastolic blood pressure significantly. However, coronary perfusion is only improved in the presence of hemodynamically relevant stenosis. An abrupt evacuation during systole results in reduction of afterload, therefore increasing left ventricular ejection fraction (LVEF) of about $10 \%$. The combination of better diastolic organ perfusion and systolic afterload reduction can improve cardiac output by up to $11 / \mathrm{min}$ in severe cardiogenic shock. The 7F IABP catheter can be easily inserted in Seldinger's technique via femoral access. Today, the majority of IABP catheters are inserted in a sheathless way. In some cases, subclavian access can also be used for IABP placement.

\section{Axial flow pumps}

The concept of continuous left ventricular unloading with an axial flow pump was introduced 25 years ago [10]. The first concept was realized with the Hemopump ${ }^{\mathrm{TM}}$ which was driven by an external motor sucking blood through a pump cannula from the left ventricle into the ascending aorta with a small impeller in the distal part of the cannula. Further technical developments included the AMED ${ }^{\mathrm{TM}}-$ System with the impeller in the descending aorta, avoiding any kinking of the drive line [11]. Today, the Impella ${ }^{\mathrm{TM}}$-System is well established in clinical routine. It consists of a pump cannula containing a micro motor and an impeller in its distal end. No external motor is necessary to provide flow rates of 2.5 and up to $5 \mathrm{l} / \mathrm{min}$ according to the pump sizes. The continuous unloading of the left ventricle establishes continuous flow into the ascending aorta and rhythm independent increase of arterial blood pressure. Especially in acute myocarditis patients, the Impella has several beneficial effects on the myocardium and the whole circulation. 


\section{Centrifugal pumps}

Centrifugal pumps allow high flow rates of more than $5 \mathrm{l} / \mathrm{min}$. While the TandemHeart ${ }^{\mathrm{TM}}$ uses a transseptal venous cannula, placed with its tip in the left atrium, other systems drain the blood through a venous cannula from the right atrium. The TandemHeart ${ }^{\mathrm{TM}}$ transfers the oxygenated blood from the left atrium through the centrifugal pump into the abdominal aorta without additional oxygenation. In case of dislocation of the pump cannula the patients are endangered of an abrupt deoxygenation.

Centrifugal pumps with an included membrane oxygenator allow biventricular support taking over completely myocardial and pulmonary functions. The blood from right atrium and cava vein is delivered by the centrifugal pump through the oxygenator back into the abdominal aorta with high flow rates after optimal gas exchange. Thus, these portable cardiopulmonary support devices (pCPS) can be also used in complete circulatory arrest. Miniaturized pCPS systems like the Lifebridge ${ }^{\mathrm{TM}}$ allow intra- or inter-hospital patient transfer with optimal oxygenation and organ perfusion. Since emergency pCPS can be inserted percutaneously, these devices are also recommended in cardiopulmonary resuscitation when other means fail to re-establish circulation. External cardiac compression devices like the Lucas ${ }^{\mathrm{TM}}$ or Autopulse ${ }^{\mathrm{TM}}-$ System may be helpful to maintain minimal circulation until the cannulas are placed through the femoral vessels by Seldinger's technique. Percutaneous cannulation allows insertion of cannula sizes between 15 and 21 French without interruption of chest compression. Coronary angiography can be performed by contra-lateral approach in Judkin's technique or brachial approach after transfer of the patient to the catheter laboratory.

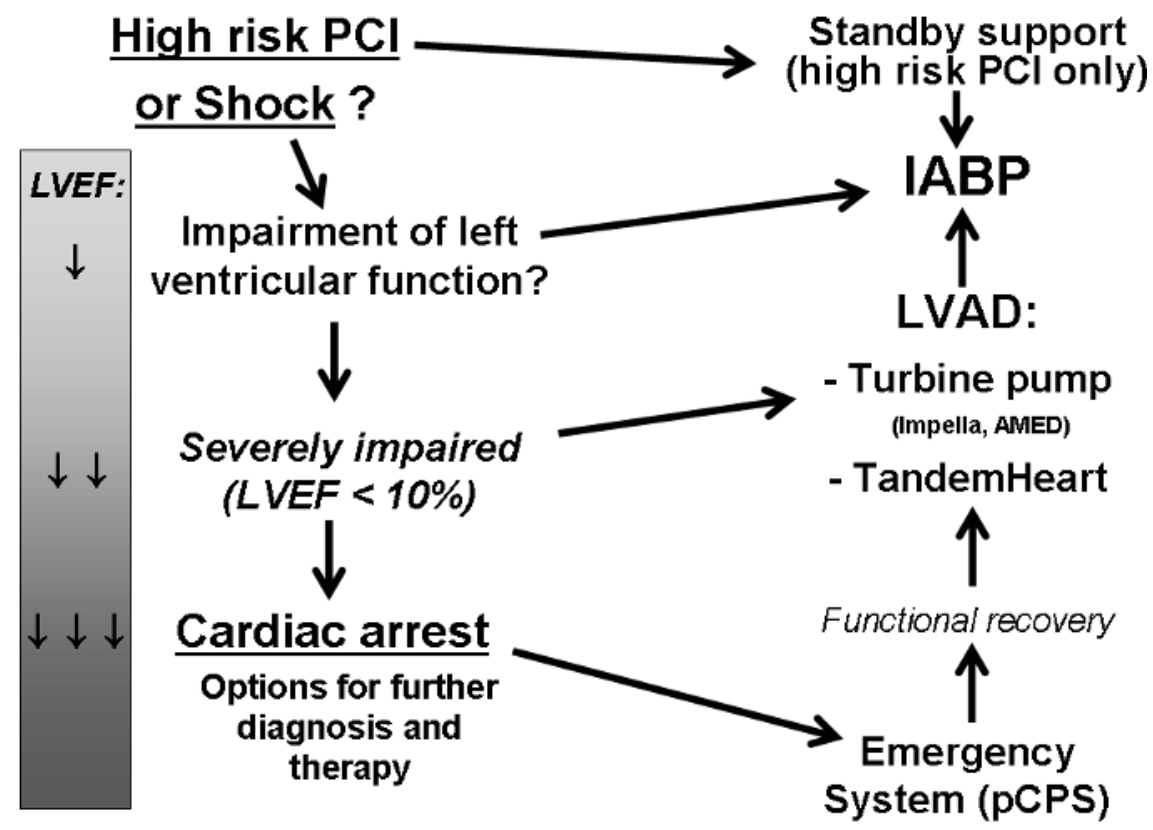

Fig. Options of percutaneous circulatory support in high risk coronary patients (mod. according to [12]). 


\section{Duration of support and weaning}

Mechanical circulatory support may be necessary in cardiogenic shock patients for several days until hemodynamic recovery. In elective high risk PCI, the device can be removed immediately after the intervention. In some cases percutaneous closure devices may be helpful. However, removal of cannulas up to diameters of 20 French is also possible under local compression and application of a compression bandage for 24 hours.

\section{Limitations and future perspectives}

Mechanical circulatory support in high risk PCI and cardiogenic shock allows hemodynamic stabilization in the acute phase especially during the intervention. Continuous sufficient organ perfusion reduces the rate of shock related MODS. However, foreign surfaces lead to artificial alteration and stimulation of blood cells causing systemic inflammatory response syndrome (SIRS). The time of mechanical circulatory support should be therefore as short as possible. Additional problems are associated with haemolysis and the need for anticoagulation. New coating techniques and optimal design of pump cannulas reduce these clinical problems. Future developments include right heart support devices and foldable systems which can be inserted through minimal diameter peripheral sheaths. After unfolding in the circulation, these devices provide powerful circulatory support [13]. Mechanical support devices are useful tools in a catheter laboratory in close cooperation with cardiac surgery. Some patients with CPR do not recover despite successful coronary revascularisation. Long term circulatory support or heart transplantation may be an option in selected cases [14]. If mechanical complications of myocardial infarction are present in the state of cardiogenic shock, further surgical therapy is recommended, too. The use of circulatory support devices also includes combinations (e.g. CPB and IABP) for a staged weaning process. Future devices may allow the combination of techniques by modification of the cannulas and pump catheters. Immunologic interventions will focus on prevention of SIRS and improved functional recovery of MODS.

\section{References}

[1] Vogel RA, Shawl F, Tommaso C, et al. Initial report of the National Registry of Elective Cardiopulmonary Bypass Supported Coronary Angioplasty. J Am Coll Cardiol 1990;15:23-29

[2] Hochman JS, Sleeper LA, Webb JG, et al. Early revascularization in acute myocardial infarction complicated by cardiogenic shock. SHOCK Investigators. Should We Emergently Revascularize Occluded Coronaries for Cardiogenic Shock. N Engl J Med 1999;341:625-634

[3] Bergelson BA, Jacobs AK, Cupples LA, et al. Prediction of risk for hemodynamic compromise during percutaneous transluminal coronary angioplasty. Am J Cardiol 1992;70:1540-1545

[4] Califf RM, Phillips HR, 3rd, Hindman MC, et al. Prognostic value of a coronary artery jeopardy score. J Am Coll Cardiol 1985;5:1055-1063

[5] Syed AI, Kakkar A, Torguson R, et al. Prophylactic use of intra-aortic balloon pump for high-risk percutaneous coronary intervention: will the Impella LP 2.5 device show superiority in a clinical randomized study? Cardiovasc Revasc Med 2011;11:91-97 
[6] Kantrowitz A. Experimental augmentation of coronary flow by retardation of the arterial pressure pulse. Surgery 1953;34:678-687

[7] Kantrowitz A, Mc KW. The experimental use of the diaphragm as an auxiliary myocardium. Surg Forum 1958;9:266-268

[8] Sanborn TA, Sleeper LA, Bates ER, et al. Impact of thrombolysis, intra-aortic balloon pump counterpulsation, and their combination in cardiogenic shock complicating acute myocardial infarction: a report from the SHOCK Trial Registry. SHould we emergently revascularize Occluded Coronaries for cardiogenic shocK? J Am Coll Cardiol 2000;36:1123-1129

[9] Sjauw KD, Engstrom AE, Vis MM, et al. A systematic review and meta-analysis of intraaortic balloon pump therapy in ST-elevation myocardial infarction: should we change the guidelines? Eur Heart J 2009;30:459-468

[10] Frazier OH, Wampler RK, Duncan JM, et al. First human use of the Hemopump, a catheter-mounted ventricular assist device. Ann Thorac Surg 1990;49:299-304

[11] Ferrari M, Aboulhosn W, Figulla HR. Successful high-risk coronary angioplasty in a patient with cardiogenic shock under circulatory assist with a 16F axial flow pump. Catheter Cardiovasc Interv 2005;66:557-561

[12] Ferrari M, Figulla HR. Circulatory assist devices in cardiology. Dtsch Med Wochenschr 2005; 130:652-656

[13] Thomas JL, Al-Ameri H, Economides C, et al. Use of a percutaneous left ventricular assist device for high-risk cardiac interventions and cardiogenic shock. J Invasive Cardiol 2010;22:360-364

[14] Ferrari M, Hekmat K, Jung C, et al. Better outcome after cardiopulmonary resuscitation using percutaneous emergency circulatory support in non-coronary patients compared to those with myocardial infarction. Acute Card Care 2011;13:30-34 


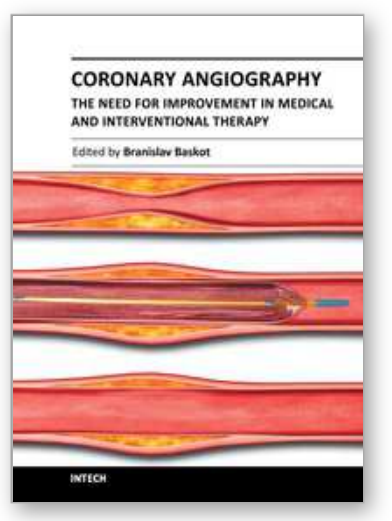

\section{Coronary Angiography - The Need for Improvement in Medical and Interventional Therapy}

Edited by Prof. Baskot Branislav

ISBN 978-953-307-641-6

Hard cover, 206 pages

Publisher InTech

Published online 06, September, 2011

Published in print edition September, 2011

In this book we examined a periprocedural complication of coronary angiography, and coronary intervention. That includes related to cardiac catheterization and diagnostic coronary angiography, and those that occur as a consequence of the specific equipment. However, improvements in devices, the use of stents, and aggressive antiplatelet therapy have significantly reduced the incident of major periprocedural complications. This book giving knowledge and experiences many of interventional cardiologists from all over the world, and provide possibility to recognize new approach in this domain. Book gives lecture on how we image and how we decide on what to treat, how to treat it, and then results of that treatment. They offer many answers to what we have today and what we will have tomorrow.

\section{How to reference}

In order to correctly reference this scholarly work, feel free to copy and paste the following:

Markus Ferrari (2011). Coronary Interventions with Mechanical Circulatory Support, Coronary Angiography The Need for Improvement in Medical and Interventional Therapy, Prof. Baskot Branislav (Ed.), ISBN: 978953-307-641-6, InTech, Available from: http://www.intechopen.com/books/coronary-angiography-the-need-forimprovement-in-medical-and-interventional-therapy/coronary-interventions-with-mechanical-circulatorysupport1

\section{INTECH}

open science | open minds

\author{
InTech Europe \\ University Campus STeP Ri \\ Slavka Krautzeka 83/A \\ 51000 Rijeka, Croatia \\ Phone: +385 (51) 770447 \\ Fax: +385 (51) 686166 \\ www.intechopen.com
}

\author{
InTech China \\ Unit 405, Office Block, Hotel Equatorial Shanghai \\ No.65, Yan An Road (West), Shanghai, 200040, China \\ 中国上海市延安西路65号上海国际贵都大饭店办公楼 405 单元 \\ Phone: +86-21-62489820 \\ Fax: +86-21-62489821
}


(C) 2011 The Author(s). Licensee IntechOpen. This chapter is distributed under the terms of the Creative Commons Attribution-NonCommercialShareAlike-3.0 License, which permits use, distribution and reproduction for non-commercial purposes, provided the original is properly cited and derivative works building on this content are distributed under the same license. 